\title{
miR-146b-5p suppresses glioblastoma cell resistance to temozolomide through targeting TRAF6
}

\author{
ZHONGRUN QIAN $^{1 *}$, SUNHAI ZHOU $^{1 *}$, ZHIYI ZHOU $^{1 *}$, XI YANG $^{1}$, SHUANLIN QUE $^{3}$, \\ JIN LAN ${ }^{1}$, YONGMING QIU ${ }^{1}$ and YINGYING LIN ${ }^{1,2}$ \\ ${ }^{1}$ Department of Neurosurgery, ${ }^{2}$ Shanghai Institute of Head Trauma, Ren Ji Hospital, School of Medicine, \\ Shanghai Jiao Tong University, Shanghai; ${ }^{3}$ Department of Neurosurgery, The First Hospital of \\ Longyan Affiliated to Fujian Medical University, Longyan, Fujian, P.R. China
}

Received March 1, 2017; Accepted August 8, 2017

DOI: $10.3892 /$ or.2017.5970

\begin{abstract}
Temozolomide (TMZ), as a kind of alkylating agent, is widely utilized for the treatment of glioblastoma (GBM). However, temozolomide resistance (TR) often develops quickly and results in tumor recurrence and poor outcome. Recent advances have demonstrated that miRNAs exert critical roles in chemoresistance. Downregulation of miR-146b-5p promotes glioma cell proliferation, reduces apoptosis, and correlates with poor survival of patients. Nonetheless, the function of miR-146b-5p in temozolomide resistance remains unclear. In the present study, we successfully generated U87 and U251-TR cells, and found that miR-146b-5p was downregulated in TR cells. Overexpression of miR-146b-5p restored sensitivity of U87/U251-TR cells to TMZ by targeting tumor necrosis factor receptor-associated factor 6 (TRAF6). The levels of TRAF6 were inversely related to miR-146b-5p levels, and overexpression of TRAF6 in miR-146b-5p-OE cells enhanced the resistance against TMZ. Moreover, temozolomide-resistant GBM cells had a higher level of phosphorylated protein kinase B (AKT) and P65. Overexpression of miR-146b-5p or TRAF6 knockdown significantly decreased the level of p-AKT and p-p65. Collectively, our data demonstrated that miR-146b-5p, as a tumor suppressor, mediated temozolomide resistance in GBM cells through negatively regulating TRAF6 expression, indicating that miR-146b-5p and its targeted genes would be potential therapeutic targets for glioma therapy.
\end{abstract}

\section{Introduction}

Glioblastoma (GBM) is the most malignant and prevalent type of primary brain malignancies $(1,2)$. These tumors are

Correspondence to: Dr Yingying Lin or Dr Yongming Qiu, Department of Neurosurgery, Ren Ji Hospital, School of Medicine, Shanghai Jiao Tong University, Room 118, Building 11, 1630 Dongfang Road, Pudong, Shanghai 200127, P.R. China

E-mail: yylin@sibs.ac.cn

E-mail: qiuzhoub@hotmail.com

Key words: glioblastoma, miR-146b-5p, temozolomide, resistance, TRAF6 not strictly focal lesions, and disseminate along the myelinated axons, blood vessels, or through the subarachnoid space (3), which makes complete resection difficult. Despite current multimodal treatments, the median survival time of GBM patients is merely 15 months from the date of diagnosis and the 5-year survival rate is $<10 \%$ (4). Clinically, the first-line therapy for GBM comprises neurosurgery with maximum feasible resection, followed by alkylating agent basedchemotherapy concomitant with and after radiotherapy $(1,4,5)$. Temozolomide, as the most frequently used alkylating agent, exhibits potent activity against malignant glioma with minimal additional toxicity. TMZ most often alkylates DNA at the position of $\mathrm{N}^{7}$ or $\mathrm{O}^{6}$ guanine residues, which induces DNA double-strand breakage and subsequent apoptosis $(6,7)$. However, most GBM patients are resistant to this alkylating agent eventually, greatly compromising the long-term tumor control. $\mathrm{O}^{6}$ methylguanine-DNA methyltransferase (MGMT), a cellular DNA repair protein, could rapidly reverse alkylation at the $\mathrm{O}^{6}$ position of guanine $(7,8)$. Although a growing number of research indicates that MGMT expression is responsible for chemoresistance to TMZ in GMB cells, TMZ-resistant (TR) GBM tissue analysis showed reduced MGMT expression in $>50 \%$ of GBM cases $(9,10)$. Therefore, it is of importance to identify the diverse molecular mechanisms involved in chemoresistance for finding ways to sensitize GBM cells to TMZ to ameliorate the therapeutic efficacy.

MicroRNAs (miRNAs or miRs) are a class of endogenous small (19-25 nucleotides) non-coding single-stranded RNAs that are involved in post-transcriptional regulation either by degrading a specific mRNA(s) or inhibiting translation (11). Through binding to the 3'-untranslated region of mRNAs, miRNAs have been proved to play crucial roles in a wide range of physiological and pathological processes, including cancer development and progression $(12,13)$. Increasing evidence showed that miRNAs are aberrantly expressed in human cancer tissues, contributing to regulating DNA damage response, interfering with response to chemotherapy as consequence $(7,14)$. For example, some studies have found that many miRNAs, such as miR-20a, miR-195, miR-181b, regulated the chemosensitivity of glioblastoma cells to TMZ (15-17). miR-146b-5p, located within 10q24-26, is a frequently missing chromosomal region in GBM (18-20). Overexpression of 
miR-146b-5p could suppress GBM cell invasion and migration by targeting MMP16, while increasing apoptosis of GBM cells (18). Furthermore, upregulation of miR-146b-5p also attenuates cell viability, stem cell marker expression and neurosphere formation (21). However, little is known about whether miR-146b-5p is involved in the regulation of the chemosensitivity of glioblastoma cells to TMZ.

TRAF6, a key member of tumor necrosis factor receptorassociated factors, plays an important role in the physiological and pathological processes (22). TRAF6 is characterized to be a signal transducer of TNFR superfamily and Toll/ interleukin-1 (IL-1) family (23). Furthermore, TRAF6 also functions as an E3 ubiquitin ligase, catalyzing K63 polyubiquitination of various proteins, such as TAK1 and legumain $(24,25)$. Elevated expression of TRAF6 has been found in colon cancer, breast cancer and lung cancer, and TRAF6 promotes proliferation of these cancer cells (25-27). Liu et al (28) previously reported that TRAF6 as a direct functional target gene regulated by miR-146b-5p promoted glioma cell invasion and suppressed apoptosis. As TMZ-resistant cells usually are characterized by increased invasiveness and declined apoptosis, the function of TRAF6 in TMZ resistance deserves to be understood.

Thus, in the present study, we investigated the expression and function of miR-146b-5p and TRAF6 in GBM cancerous tissues and TMZ-resistant cell lines. Our results indicated that miR-146b-5p was down regulated in TMZ-resistant cell lines, and TRAF6 was a direct functional target of miR-146b-5p. By upregulation of miR-146b-5p or silencing TRAF6 can sensitize GBM cells to TMZ. Our studies indicate that miR-146b-5p and TRAF6 may be potential therapeutic targets for GBM treatment.

\section{Materials and methods}

Cell culture. The human GBM cell lines U87-MG and U251-MG (purchased from the Cell Bank of the Shanghai Branch of the Chinese Academy of Sciences, 2014) were cultured in Dulbecco's modified Eagle's medium (Invitrogen, USA) supplemented with 10\% fetal bovine serum (Invitrogen) and maintained in a humidified atmosphere with 5\% carbon dioxide-humidified atmosphere at $37^{\circ} \mathrm{C}$.

Patients and tissue samples. The study was approved by the Ethics Committee of the Ren Ji Hospital, School of Medicine, Shanghai Jiao Tong University. Written consent was obtained from patients or guardians on behalf of the minors enrolled in the study. Six GBM patients receiving standard chemoradiotherapy at Ren Ji Hospital, Shanghai Jiao-Tong University between January 2012 and December 2013 were recruited and followed for this study. The initial survival time of patients with primary GBM after standard chemoradiotherapy of less than six months was designated as resistant to TMZ treatment and the initial survival time greater than ten months was designated as sensitive to TMZ.

Generation of temozolomide-resistant glioblastoma cells. The parental U87-MG and U251-MG cells were treated with temozolomide at $400 \mu \mathrm{M}$ or DMSO solvent control at a final concentration of $0.1 \%$ for 4 weeks. In brief, to generate
TMZ-resistant GBM cells, cells were treated with fresh TMZ every day for 5 consecutive days and then exposed to the fresh TMZ every 3 days to a total of 3 weeks. Survival cells were continued its culture for 4 weeks until deriving stable resistant cell lines (TMZ-resistant U251 cells (U251-TR) and U87 cells (U87-TR) for subsequent experiments.

Western blot analysis. To analyze the expression of proteins, western blot assays were performed using the following primary antibodies: rabbit anti-human TRAF6 (1:1,000 dilution, ab33915; Abcam, USA), AKT (1:1,000 dilution, 9272; Cell Signaling Technology), p-AKT (1:1,000 dilution, 4060; Cell Signaling Technology), P65 (1:1,000 dilution, 8242; Cell Signaling Technology), p-p65 (1:1,000 dilution, 3033; Cell Signaling Technology) and mouse anti-actin (1:10,000; Millipore). Briefly, tissues were lysed with RIPA buffer [50 mM Tris- $\mathrm{HCl}$ (pH 7.5), $150 \mathrm{mM} \mathrm{NaCl,} 1 \%$ Triton X-100, $0.5 \%$ Na-deoxycholate] containing protease inhibitors (Complete, Mini; Roche); 20-30 $\mu \mathrm{g}$ samples of the lysates were separated on 8-12\% SDS-PAGE gels and transferred to PVDF membranes. The membranes were incubated with primary antibodies overnight at $4^{\circ} \mathrm{C}$. The primary antibody incubation was followed by incubation with an HRP-conjugated secondary antibody. The bound antibodies were detected using an ECL kit (PI32209; Pierce).

cDNA synthesis and real-time PCR reaction. Total RNA was extracted from cells using TRIzol reagent (Life Technology, USA). After that, mRNA was reverse transcribed using SuperScript First-Strand Synthesis-System for RT-PCR (Invitrogen) according to the manufacturer's instructions. Real-time PCR was performed using SYBR Green reagents (Takara, Japan) containing $100 \mu \mathrm{M}$ final concentration of primers (primer sequences were listed in Table I). PCR conditions were: $95^{\circ} \mathrm{C}$ for $15 \mathrm{sec}$, followed by 40 cycles of $95^{\circ} \mathrm{C}$ for $5 \mathrm{sec}, 60^{\circ} \mathrm{C}$ for $30 \mathrm{sec}$. PCRs were performed in triplicates using the Roche LightCycler $480^{\circledR}$ RT-PCR system (Roche).

Lentivirus-mediated gene knockdown and overexpression. The target sequences for TRAF6 siRNAs were 5'-GCTCTTGG TGGATCATCAA-3' (TRAF6-KD1) and 5'-GCATGTTCAAT GGGAGCTTGGA-3' (TRAF6-KD2). After $48 \mathrm{~h}$, the efficiency of TRAF6 knockdown was confirmed via quantitative real-time polymerase chain reaction (qRT-PCR) and western blot analysis.

Lentiviral vectors for human TRAF6-shRNA carrying a green fluorescent protein (GFP) sequence were constructed by Hanyin Co. (Shanghai, China). The recombinant TRAF6 knockdown lentivirus and the negative control (NC) lentivirus (GFP-lentivirus; Hanyin Co. Shanghai, China) were prepared and titered to $10^{9} \mathrm{TU} / \mathrm{ml}$ (transfection unit). To obtain the stable TRAF6-knockdown cell line, cells were seeded in 6-well dishes at a density of $2 \times 10^{5}$ cells per well. The cells were then infected with the same titer virus with $8 \mu \mathrm{g} / \mathrm{ml}$ polybrene the following day. Approximately $72 \mathrm{~h}$ after viral infection, GFP expression was confirmed under a fluorescence microscope, and the culture medium was replaced with selection medium containing $4 \mu \mathrm{g} / \mathrm{ml}$ puromycin. The cells were then cultured for $\geq 14$ days. The puromycin-resistant cell clones were isolated, amplified in medium containing $2 \mu \mathrm{g} / \mathrm{ml}$ puromycin for 
Table I. Sequences of qPCR primers.

\begin{tabular}{ll}
\hline Primer name & \multicolumn{1}{c}{ Sequence } \\
\hline Human-TRAF6-F & 5'-TGATGTAGAGTTTGACCCACCC-3' \\
Human-TRAF6-R & 5'-GTCAACTGGACATTTGTGACCTG-3' \\
hsa-miR146b-5p & TGAGAACTGAATTCCATAGGCT
\end{tabular}

7-9 days, and transferred to a medium without puromycin. The clones were designated as TRAF6-KD or NC cells. TRAF6 was cloned into a pMSCV-IRES-GFP vector. For overexpression of TRAF6, GBM cells were transfected by viral supernatant from 293T cells transfected with TRAF6 or control vector. The clones were designated as TRAF6-OE or NC cells.

CCK8. A CCK8 assay was conducted according to the kit instructions (DH343-2, Beijing Dongsheng Biotechnology, China). Briefly, cells were plated at equal cell density $(2,000$ cell $/ 100 \mu \mathrm{l}$ per well) in 96-well plates with cetuximab $(300 \mu \mathrm{g} / \mathrm{ml})$ for continuous detection over a 5-day period. At the beginning of the second day, the culture was terminated by adding $10 \mu \mathrm{l}$ CCK8 $(5 \mathrm{mg} / \mathrm{ml})$ to the original culture medium. After $2 \mathrm{~h}$, the plates were measured using a microplate reader (Elx800; Biotek, USA). Cell proliferation was measured using OD490 values.

Construction of luciferase reporter vectors. The TRAF6 gene 3'-UTR was generated by inserting the 3' flanking region of the TRAF6 gene into pGL3-basic vector (Promega, Fitchburg, WI,USA). To identify the critical miRNA binding region of the TRAF6, subsequently, mutated luciferase reporter constructs were generated by PCR. All the constructs were verified by sequencing to rule out the possibility of any PCR error.

Transient transfections and luciferase assays. Cells were seeded in 96-well plates and grown to a density of $80 \%$ confluence before transfection. Each luciferase reporter construct (100 ng) and $0.3 \mu \mathrm{l}$ Lipofectamine ${ }^{\mathrm{TM}} 2000$ (Invitrogen) reagent in Opti-MEM were transfected according to the manufacturer's instructions. In order to correct transfection efficiency, additional $4 \mathrm{ng}$ of the pRL-TK vector which contained the Renilla luciferase gene (hRluc) under the control of the Herpes simplex virus thymidine kinase promoter was co-transfected in each well. The cells were harvested after 48 -h transfection, and then lysed with $20 \mu$ l passive lysis buffer for each well (Promega). Luciferase activity was measured using Dual-Luciferase Reporter assay system (Promega) on a luminescence reader (Acuu FLEX Lumi 400; Aloka, Tokyo, Japan).

The nude mouse tumor xenograft model. For brain xenografts, briefly, $1 \times 10^{6}$ U87-TR cells with or without miR-146b-5p were stereotactically injected into the brains of the 6-week-old athymic nude mouse. A 1-mm drill was then used to make a burr hole $1 \mathrm{~mm}$ anterior of the bregma and $2 \mathrm{~mm}$ to the right of the skull. A 21-gauge Hamilton syringe was advanced $3.5 \mathrm{~mm}$ deep, and then retracted $3 \mathrm{~mm}$ for implanting $5 \mu \mathrm{l}$ of tumor cell suspension. After 1 week, half of the mice in each group were treated by TMZ by tail vein injection. Mice were monitored for the development of tumors by magnetic resonance imaging (MRI). The animal study was approved by the Institutional Animal Care and Use Committee of Renji Hospital, Shanghai Jiao Tong University.

Statistical analysis. All statistical analyses were performed using SPSS for Windows v.17.0 (SPSS, Chicago, IL, USA). All results were considered significant at two-sided $\mathrm{p}<0.05$ value.

\section{Results}

TRAF6 was upregulated in TMZ-resistant GBM cells and tissues. We established TMZ-resistant GBM cells (U87-TR and U251-TR) with gradually elevated TMZ concentration in wild-type GBM cells (U87 and U251). The $\mathrm{IC}_{50}$ value of resistant cells increased nearly 5-fold compared with parental wild-type cells (Fig. 1A and B). The western blotting results showed that TRAF6 expression was upregulated in both TMZ-resistant GBM cells (Fig. 1C and D). Further we collected specimens from GBM patients with different response to TMZ treatment. The immunohistochemistry staining results showed that the expression of TRAF6 was higher in TMZ-resistant GBM tissues than that of TMZ sensitive GBM tissues (Fig. 1E). Taken together, these results suggest that TRAF6 was upregulated in TMZ-resistant GBM cells and cancerous tissues.

Knockdown of TRAF6 in TMZ-resistant GBM cells reduced the TMZ resistance. We then knocked down TRAF6 in U87-TR and U251-TR cells. Both the RT-PCR and western blotting results indicated that TRAF6 were efficiently suppressed by shRNAs (Fig. 2A-D). The CCK8 analysis revealed that knockdown of TRAF6 significantly reduced cell proliferation ability (Fig. 2E and F). CCK8 assay of $\mathrm{IC}_{50}$ of these cells showed that knockdown of TRAF6 in TMZ-resistant GBM cells reduced the TMZ resistance (Fig. $2 \mathrm{G}$ and $\mathrm{H}$ ).

Downregulated miR-146b-5p targeted TRAF6 in temozolomide-resistant GBM cells. We further searched for the upstream regulator of TRAF6 in TMZ-resistant GBM cells. Analyzing the potential miRNA binding to the 3'-UTR of TRAF6 by TargetScan found that there were three binding motifs to miR-146b-5p, suggesting that miR-146b-5p might be the regulator of TRAF6 (Fig. 3A). According to the results of luciferase reporter assay, overexpression of miR-146b-5p inhibited the luciferase activity while mutated the conserved binding sequences of miR-146b-5p, miR-146b-5p failed to inhibit the luciferase activity in U87-TR and U251-TR cells (Fig. 3B and C). The overexpression of miR-146b-5p was validated by RT-PCR in two cell lines (Fig. 3D). Consistent with these results, the mRNAs of TRAF6 were downregulated in miR-146b-5p overexpressed U87-TR and U251-TR cells (Fig. 3E). The western blotting results also showed that TRAF6 was reduced in miR-146b-5p overexpressed U87-TR and U251-TR cells (Fig. 3F). Finally the expression of miR-146b-5p was examined in GBM wild-type cells and TMZ-resistant cells. The RT-PCR results showed that the expression of miR-146b-5p was downregulated in U87-TR and U251-TR cells (Fig. 3G and H). Thus, all of these results demonstrated that downregulated miR-146b-5p targeted TRAF6 in temozolomide-resistant GBM cells. 

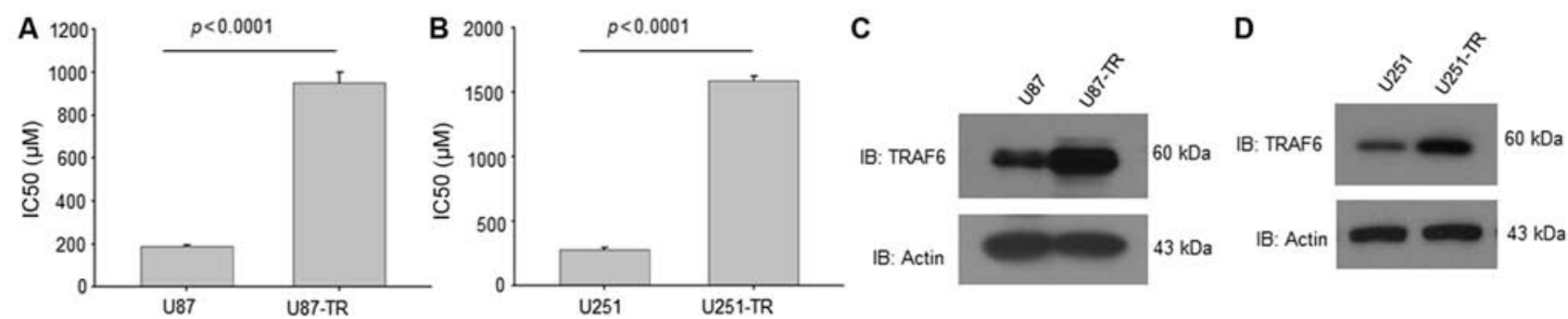

E

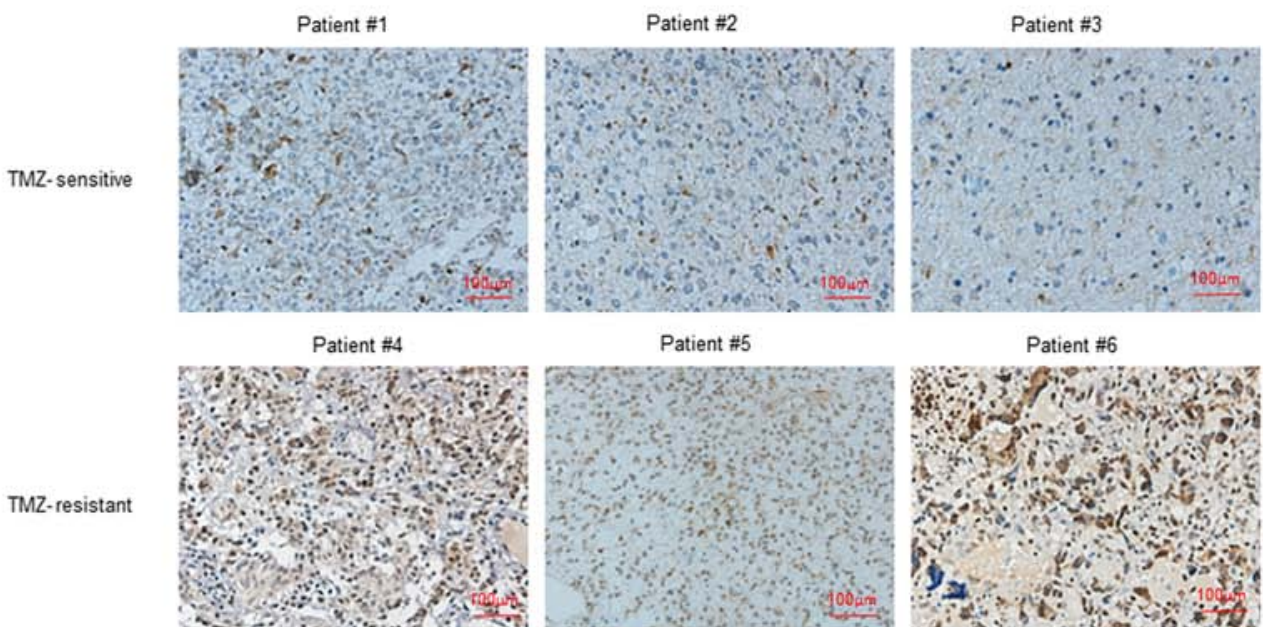

Figure 1. TR GBM TMZ-resistant cells and tissue expressed a high level of TRAF6. (A and B) The $\mathrm{IC}_{50}$ of TR cells is greater than wild-type U87 and U251 cells, indicating successful generation of TR cells. Data represents the mean value \pm SD of triplicate cultures, $p<0.0001$. (C and D) Western blot analyses for expression of TRAF6 in TMZ-resistant cells and control group. (E) Immunohistochemical staining showed TRAF6 was overexpressed in TMZ-resistant patients compared to TR-sensitive patients. All data are representative of three independent experiments. GBM, glioblastoma; TRAF6, tumor necrosis factor receptor associated factor $6 ; \mathrm{IC}_{50}$, half maximal inhibitory concentration; TR, temozolomide-resistant; TMZ, temozolomide.
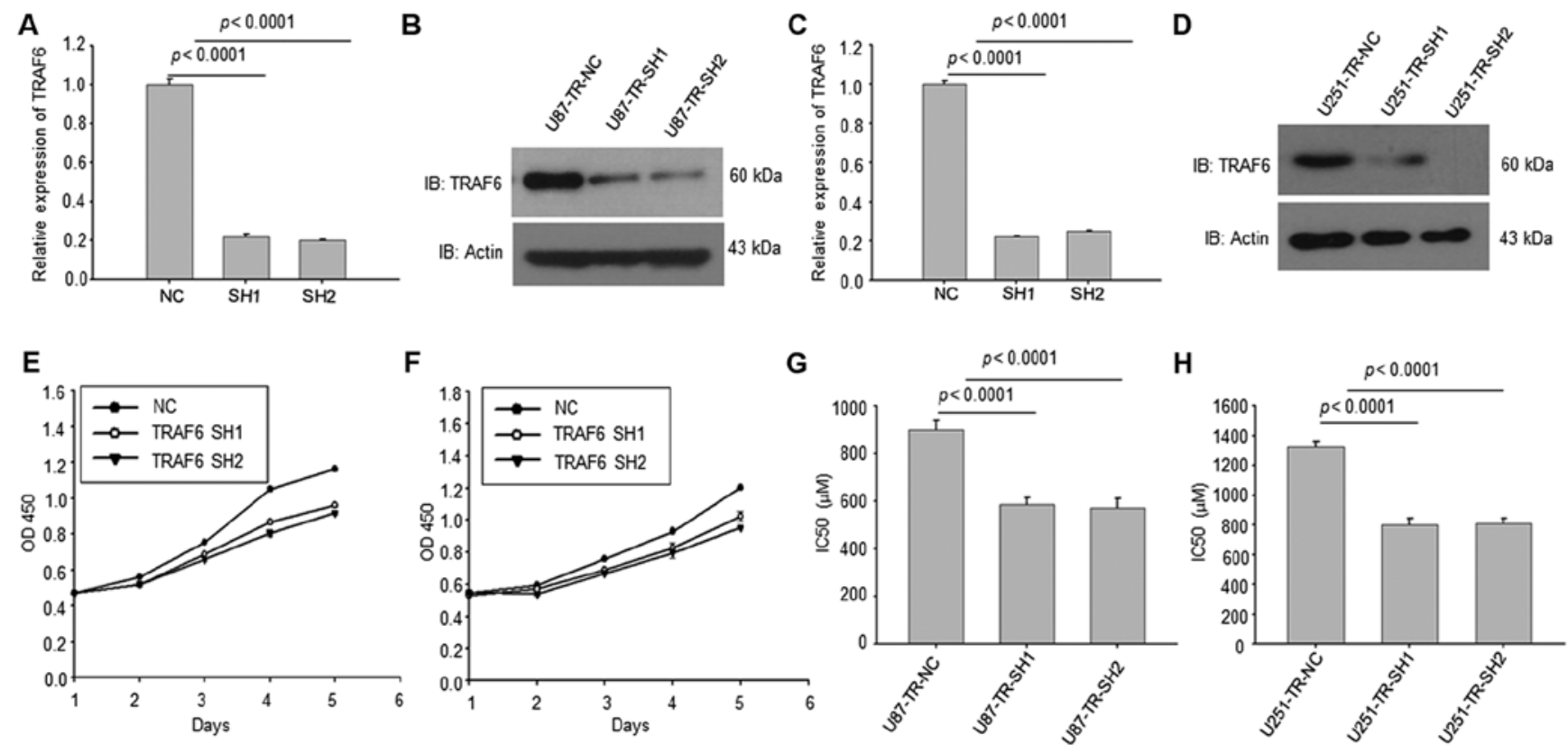

Figure 2. TRAF6 knockdown reverses sensitivity of TR cells to TMZ. (A-D) By transfecting shRNAs into TR cells, western blot analysis and RT-PCR results showed mRNA and protein level of TRAF6 decreased efficiently compared to control group, $\mathrm{p}<0.0001$. (E and F) The CCK8 analysis revealed that knockdown of TRAF6 reduced cell proliferation ability. $\left(\mathrm{G}\right.$ and $\mathrm{H}$ ) With the knockdown of TRAF6 in TR cells, the $\mathrm{IC}_{50}$ value decreased obviously compared to control group. All experiments were performed in triplicate and the data in $(\mathrm{A}),(\mathrm{C})$ and $(\mathrm{E}-\mathrm{H})$ are presented as mean $\pm \mathrm{SD}, \mathrm{p}<0.0001$. TRAF6, tumor necrosis factor receptor associated factor 6; TR, temozolomide-resistant; TMZ, temozolomide; shRNAs, short hairpin RNA; RT-PCR, real-time polymerase chain reaction; CCK8, cell counting kit-8; $\mathrm{IC}_{50}$, half maximal inhibitory concentration. 
A
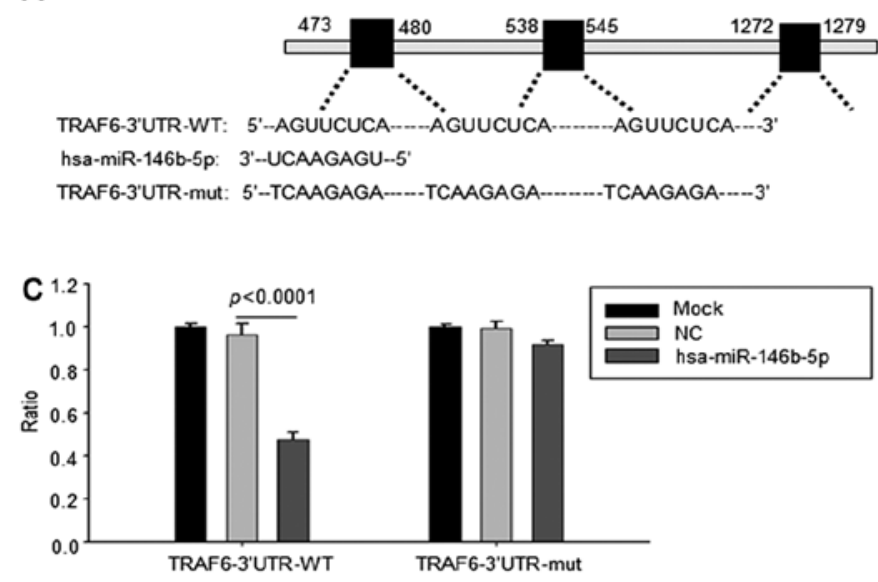

E

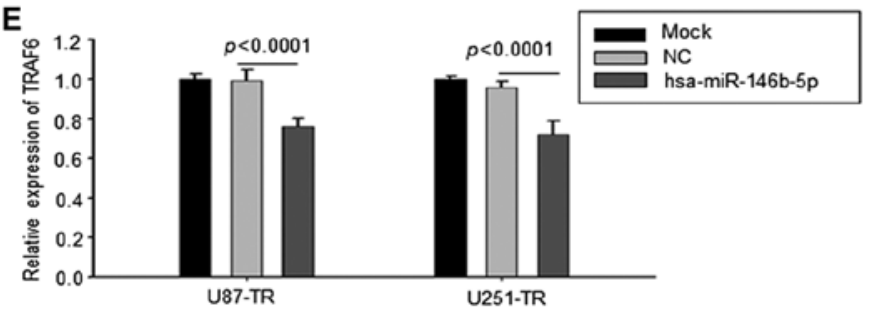

G

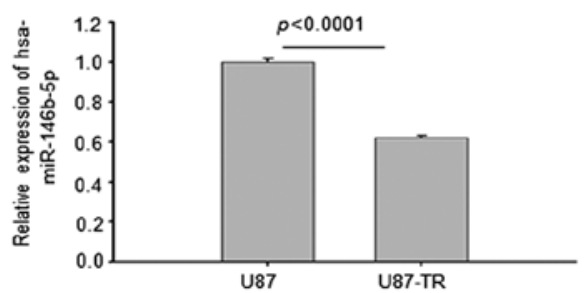

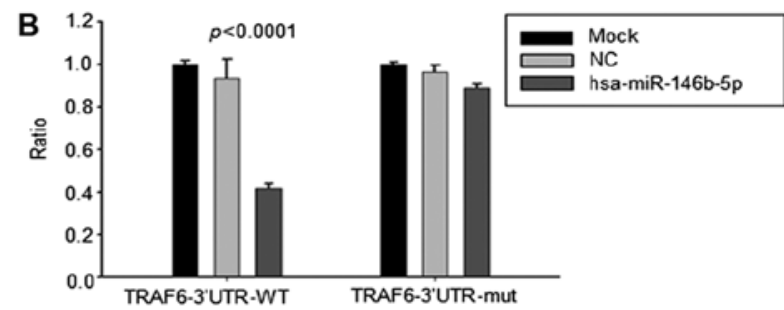

D

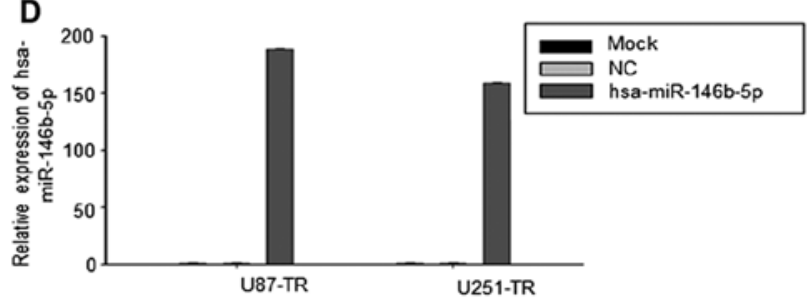

$\mathbf{F}$

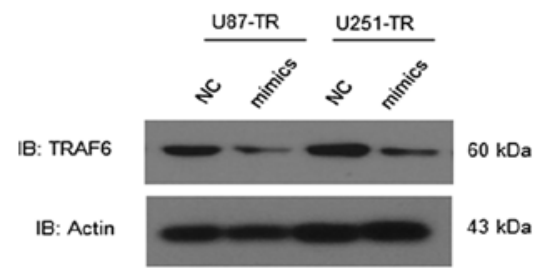

H

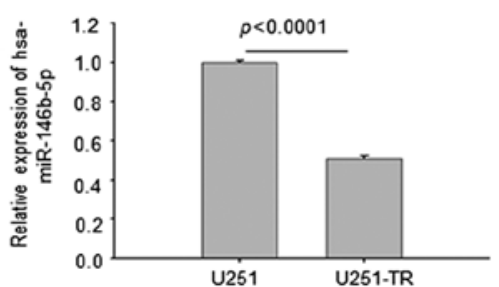

Figure 3. TRAF6 was a direct target gene of miR-146b-5p in TR cells. (A) Three potential miR-146b-5p binding sites in TRAF6 3'-UTR predicted with TargetScan. (B and C) Luciferase reporter assay was conducted to confirm that TRAF6 3'-UTR luciferase activity is inhibited in U87-TR and U251-TR cells by transfection with hsa-miR-146b-5p lentivirus. Expression of miR-146b-5p was detected in these two cells by RT-PCR, p<0.0001. (D-F) RT-PCR and western blotting assays showed expression of TRAF6 was inhibited in TR cells, p<0.0001. (G and H) The RT-PCR results showed that the expression of miR-146b-5p was downregulated in U87-TR and U251-TR cells, $\mathrm{p}<0.0001$. All experiments were performed in triplicate and the data in (B-E, G and H) are presented as mean \pm SD. TRAF6, tumor necrosis factor receptor associated factor 6; TR, temozolomide-resistant; UTR, untranslated regions; RT-PCR, real-time polymerase chain reaction.

was overexpressed in U87-TR and U251-TR cells (Fig. 4A and B). The CCK8 analysis revealed that overexpression of miR-146b-5p significantly reduced cell proliferation ability (Fig. 4C and D). Moreover, the comparison of $\mathrm{IC}_{50}$ showed that overexpression of miR-146b-5p in TMZ-resistant GBM cells reduced the TMZ resistance (Fig. $4 \mathrm{E}$ and $\mathrm{F}$ ). These results revealed that $\mathrm{miR}-146 \mathrm{~b}-5 \mathrm{p}$ was a negative regulator of $\mathrm{TMZ}$ resistance in GBM cells.

Overexpression of TRAF6 in TMZ-resistant miR-146b-OE GBM cells elevated the $I C_{50}$ of TMZ. As we have found that miR-146b-5p targeted TRAF6 in TMZ-resistant GBM cells, we overexpressed TRAF6 in TMZ-resistant miR-146b-OE GBM cells. The western blotting result showed that TRAF6 expression was elevated in these cells compared to the NC control (Fig. 5A). The CCK8 analysis revealed that overexpression of TRAF6 recued the cell proliferation ability in miR-146b-5p-OE cells (Fig. 5B and C). Calculation of the $\mathrm{IC}_{50}$ of these cells showed that overexpression of TRAF6 in miR-146b-5p-OE GBM cells regained the TMZ resistance (Fig. 5D and E). Thus, these data revealed that downregulated
miR-146b-5p increased TMZ-resistance might partially be through targeting TRAF6.

miR-146b-5p enhances the chemo-sensitivity of TMZ in vivo. To assess the functions of miR-146b-5p in GBM chemo-sensitivity in vivo, an orthotopic xenotransplanted GBM model was employed. U87-TR cells overexpressing miR-NC or miR-146b-5p were stereotactically injected into the brain of the athymic nude mouse, respectively. After 1 week, the miR-NC group was randomly divided into two groups: miR-NC and miR-NC+TMZ. TMZ was delivered to the miR-NC+TMZ group by tail vein injection. The same procedure was also applied to the miR-146b-5p overexpression group. When a few mice started to show signs of morbidity, mice in each experimental group were assessed by MRI to confirm intracranial tumor formation. The survival times of every mouse of each group were recorded and analyzed by the Kaplan-Meier method. The results demonstrated that with TMZ treatment, mice in the miR-146b-5p-overexpressing group survived longer than the control group (Fig. 6). These data demonstrated that miR-146b-5p enhances the sensitivity of TMZ chemotherapy of GBM cells in vivo. 

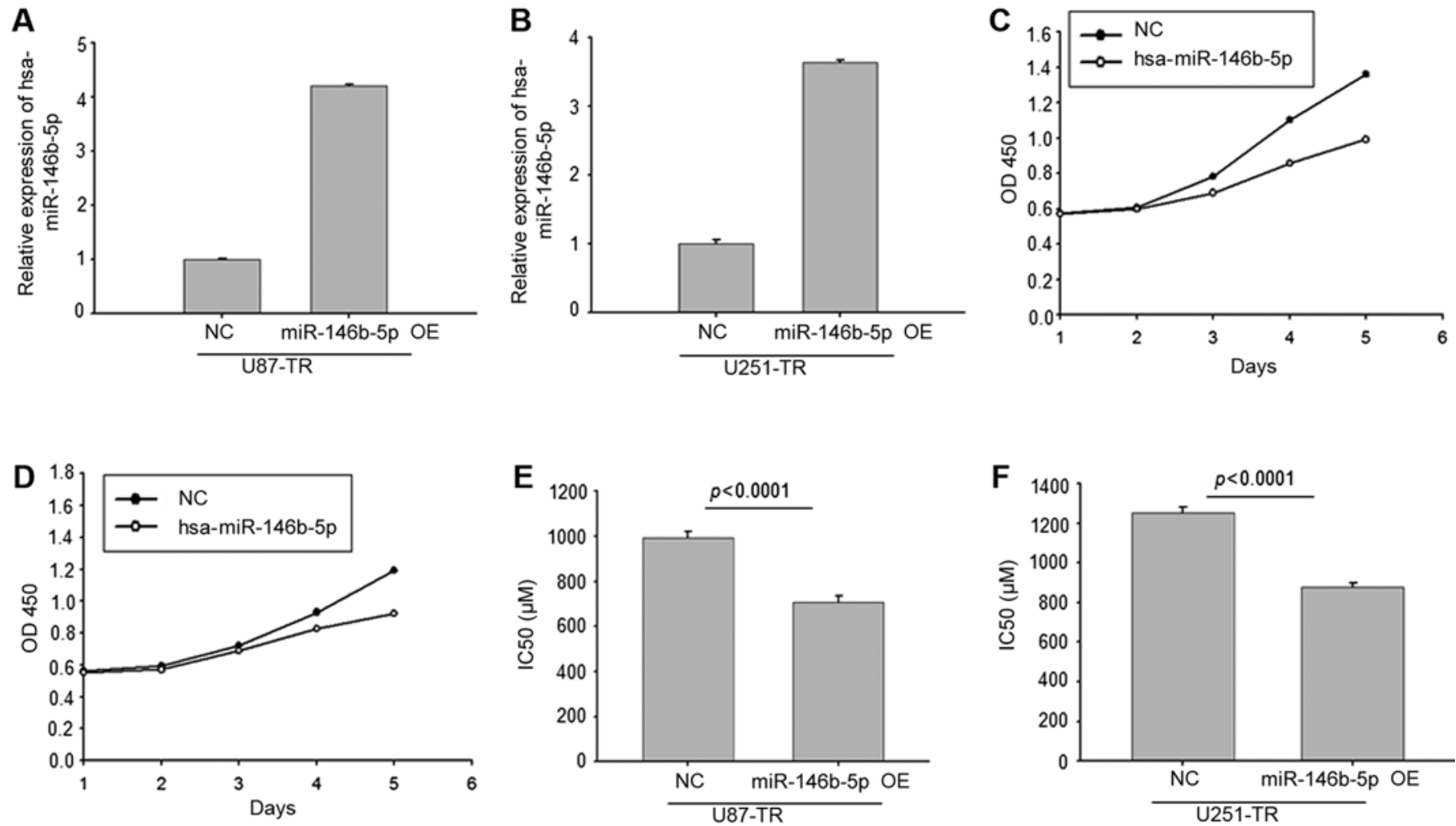

Figure 4. Upregulation of miR-146b-5p in TR cells enhanced sensitivity to TMZ. (A and B) RT-PCR results showed transfection with miR-146b-5p lentivirus significantly increased the expression of miR-146b-5p in U87 and U251-TR cells, p<0.0001. CCK8 showed cell proliferation ability was inhibited in U87-TR cells (C) and U251-TR cells (D) with the overexpression of miR-146b-5p. Introduction of miR-146b-5p lentivirus significantly reduced IC ${ }_{50}$ of U87-TR (E) and U251-TR (F) cells, p<0.0001. All data represent the mean value \pm SD of triplicate cultures. TR, temozolomide-resistant; TMZ, temozolomide; RT-PCR, real-time polymerase chain reaction; $\mathrm{CCK} 8$, cell counting kit-8; $\mathrm{IC}_{50}$, half maximal inhibitory concentration.
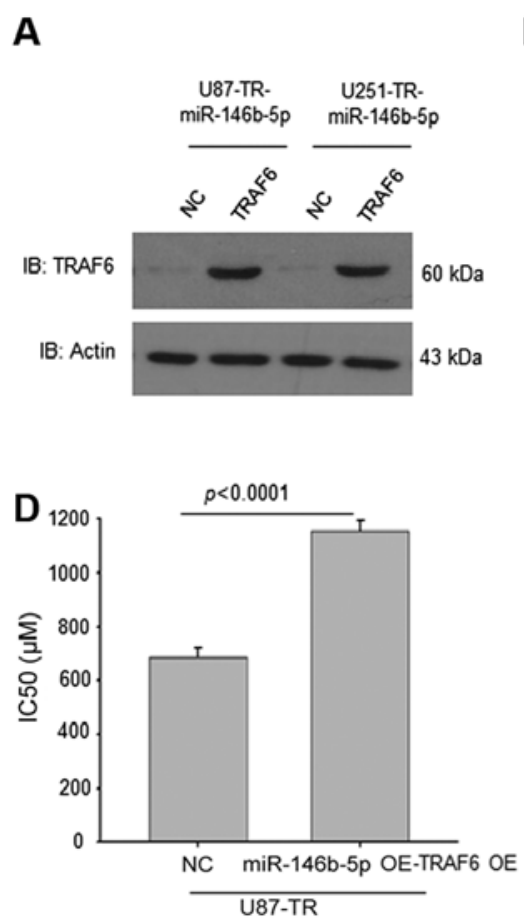
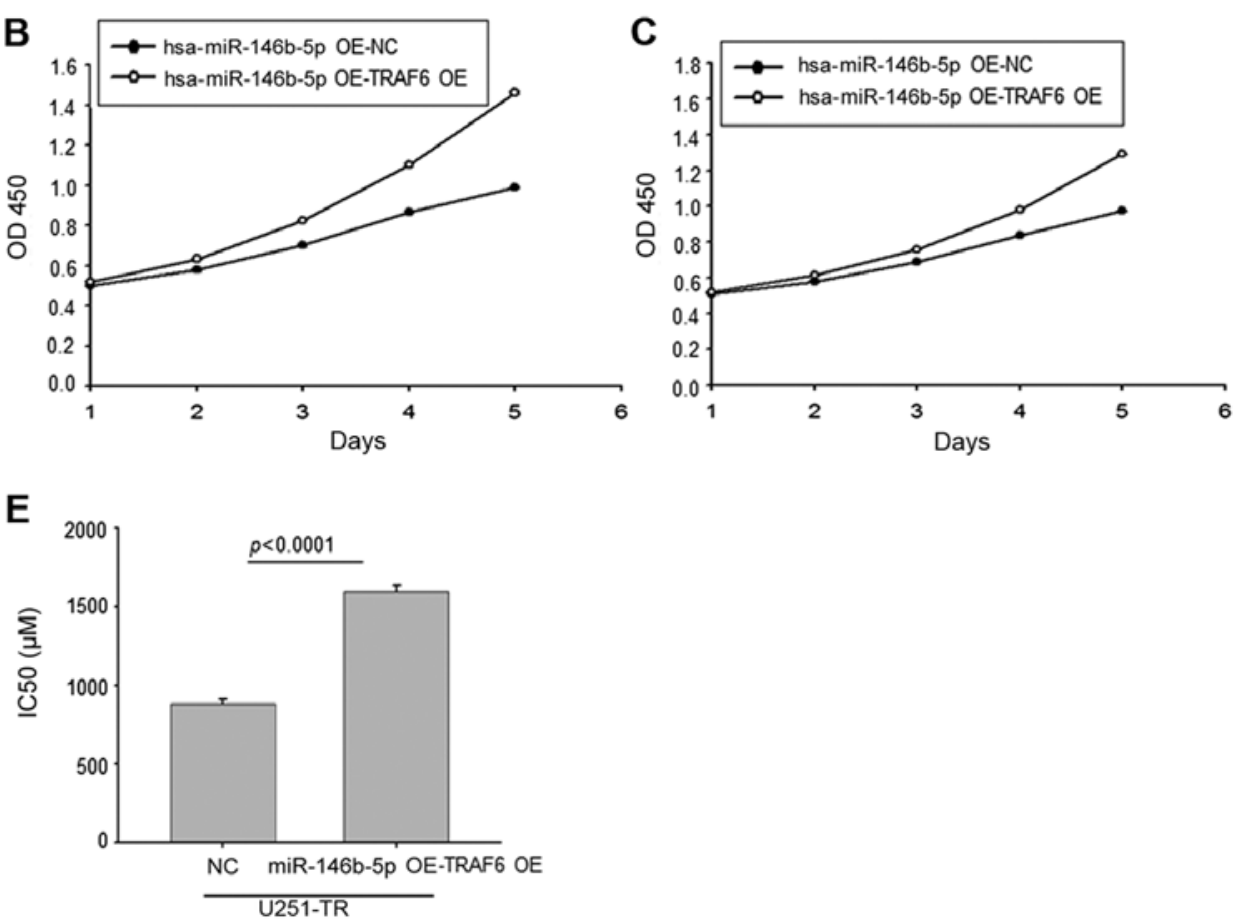

Figure 5. Overexpression of TRAF6 inhibited sensitivity of miR-146b-5p-OE TR cells to TMZ. (A) By transfection with overexpressed TRAF6 lentivirus, the level of TRAF6 protein in TMZ-resistant miR-146b-5p-OE GBM cells and control group were analyzed by western blot analysis. Transfection with overexpressed TRAF6 lentivirus, cell proliferation ability was enhanced in TMZ-resistant miR146b-OE U87 cells (B) and U251 cells (C) in CCK8 assay. (D and E) Value of $\mathrm{IC}_{50}$ showed that overexpression of TRAF6 made miR-146b-5p-OE GBM cells regain the TMZ resistance, p<0.0001. All experiments were performed in triplicate and the results in (B-E) are presented as mean \pm SD. TRAF6, tumor necrosis factor receptor associated factor 6; OE, overexpression; TR, temozolomide-resistant; TMZ, temozolomide; CCK8, cell counting kit-8; $\mathrm{IC}_{50}$, half maximal inhibitory concentration. 


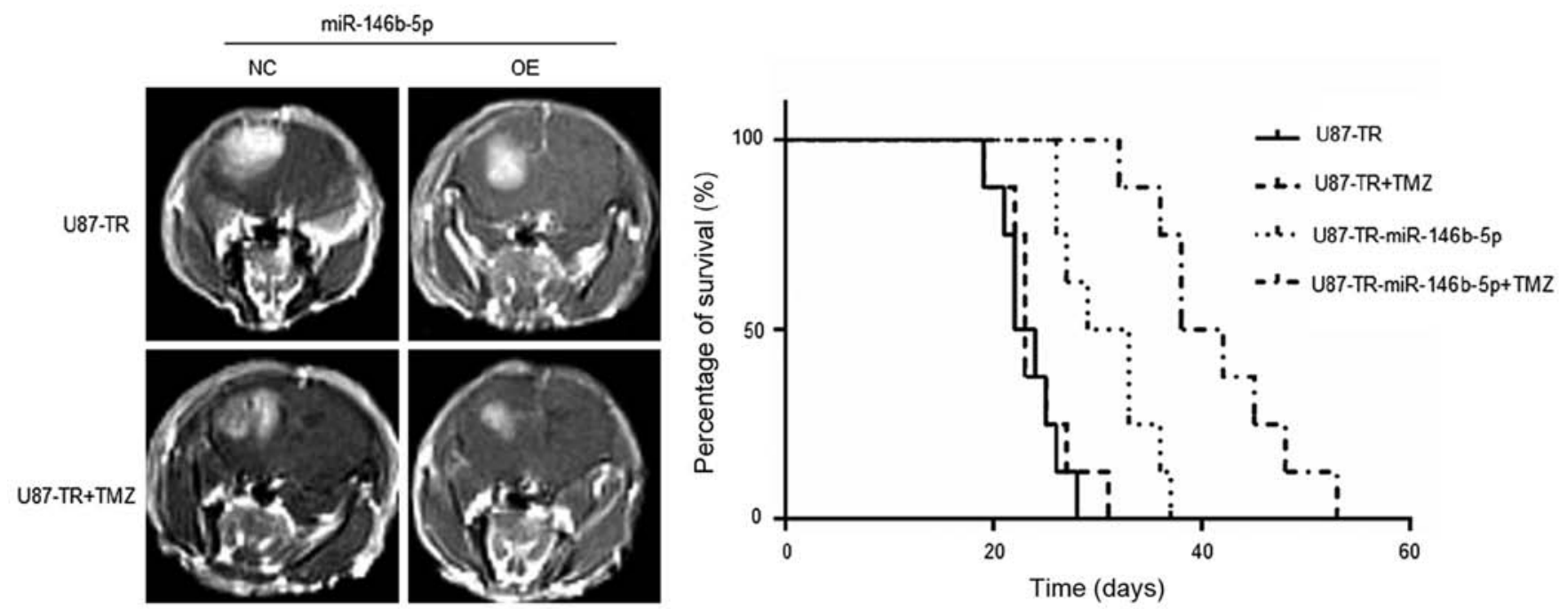

Figure 6. miR-146b-5p enhances the chemo-sensitivity of TMZ in vivo. Representative MR images of the GBM tumors orthotopically inoculated with U87-TR or U87-TR-miR-146b-5p treated with or without TMZ. Time of death was recorded as days after the GBM cell implantation.

A

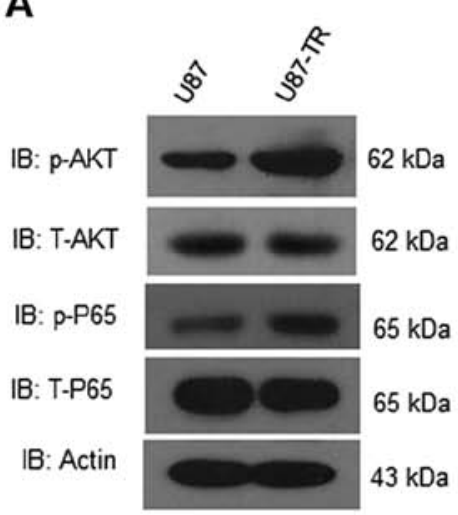

D

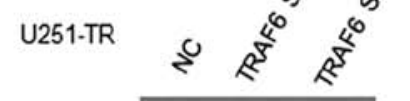

IB: p-AKT $62 \mathrm{kDa}$

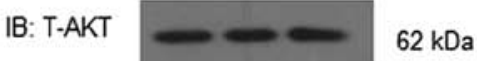

IB: p-P65

IB: T-P65

IB: Actin
B

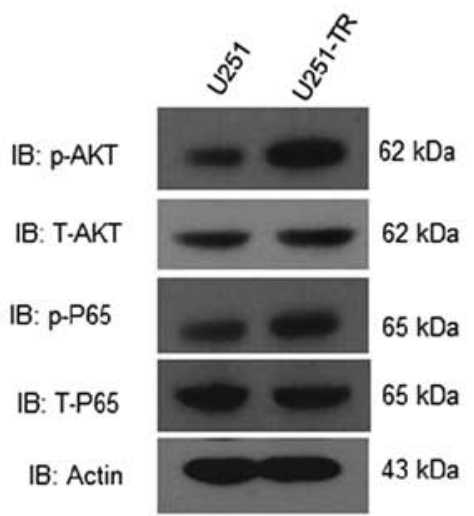

E

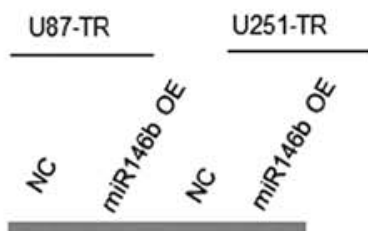

IB: p-AKT

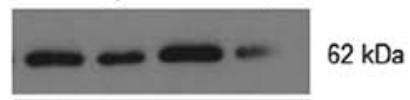

IB: T-AKT

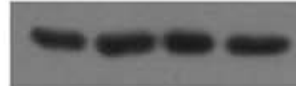

1B: p-P65

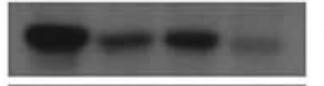

$62 \mathrm{kDa}$

$65 \mathrm{kDa}$

IB: T-P65

IB: Actin

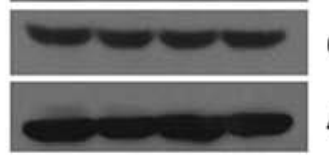

C

U87-TR

IB: p-AKT

IB: T-AKT

IB: p-P65

IB: T-P65

IB: Actin
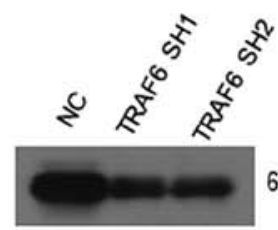

$62 \mathrm{kDa}$
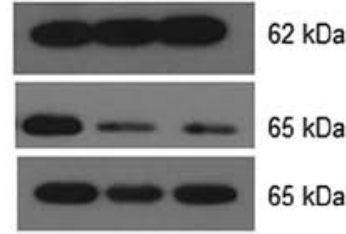

$43 \mathrm{kDa}$

F

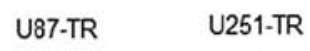

-miR146b OE -miR146b OE

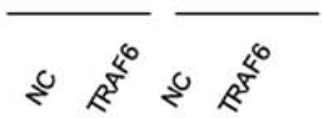

IB: p-AKT

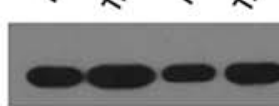

$62 \mathrm{kDa}$

IB: T-AKT $202 \mathrm{kDa}$

IB: p-P65 $=65 \mathrm{kDa}$

IB: T.P65 $=65 \mathrm{kDa}$

IB: Actin $=43 \mathrm{kDa}$

Figure 7. miR-146b-5p and TRAF6 knockdown suppress the activation of AKT/NF-кB pathway. (A and B) Western blot analyses of p-AKT, T-AKT, p-P65, T-P65 in U87, U87-TR, U251, U251-TR cells. (C and D) Expression of p-AKT, T-AKT, p-P65, T-P65 in U87/U251-TR cells and TRAF6 knockdown U87/ U251-TR cell were analyzed by western blot analysis. (E) Above proteins were analyzed in miR-146b-5p-OE U87/U251-TR cells and control group by western blot analysis. (F) With overexpression of TRAF6 in miR-146b-5p-OE U87/U251-TR cells, relative proteins of AKT/NF-kB pathway were activated. Data are

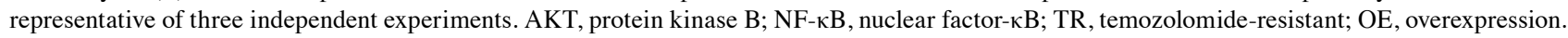

miR-146b-5p/TRAF6 regulates $A K T$ and $N F-\kappa B$ pathway activation in TMZ-resistant GBM cells. TRAF6 has been found regulating the AKT and NF- $\mathrm{BB}$ pathway in cancer.
We analyzed both pathway in TMZ-resistant GBM cells. Compared to the wild-type, phosphorylated Akt and p65 were increased in U87-TR and U251-TR cells (Fig. 7A and B). 
Knockdown of TRAF6 in U87-TR and U251-TR cells reduced the activation of Akt and p65 (Fig. 7C and D). Moreover, overexpression of miR-146b-5p in U87-TR and U251-TR cells consistently reduced the activation of Akt and p65 (Fig. 7E), and overexpression of TRAF6 in miR-146b-5p-OE TR cells would reverse this effect (Fig. 7F). Altogether, miR-146b-5p/ TRAF6 regulates AKT and NF- $\mathrm{AB}$ (nuclear factor- $\kappa \mathrm{B}$ ) pathway activation in TMZ-resistant GBM cells.

\section{Discussion}

The regimen of postoperative usage of temozolomide combined with radiotherapy has become the standard therapy for GBM, which significantly improves the overall survival for patients with glioma (1). However, TMZ resistance rapidly progresses among most patients, becoming the main reason why treatment fails and tumor recurs. Besides the critical role of MGMT in enhancing TMZ resistance, other mechanisms mediating intrinsic or acquired resistance to $\mathrm{TMZ}$ have also been recognized. As previous studies reported, epithelial-tomesenchymal transition, re-organization of the cytoskeleton, activation of pro-survival pathway genes, disturbance of the mismatch repair system, autophagy, glioma stem cells, and abnormal expression of microRNAs were closely associated with temozolomide resistance $(10,15-17,29-34)$. In the present study, we acquired a stable TMZ-resistant cell line, in which miR-146b-5p was significantly downregulated and upregulated expression can sensitize GBM cells to TMZ.

MicroRNAs have been found to play critical oncogenic roles in tumor development and drug resistance. Some previous studies have reported the relationship between miRNAs and TMZ resistance in GBM. Overexpression of miR-17, miR-20a, and miR-181b sensitizes the glioma cells to TMZ-induced cytotoxicity, while upregulation of miR-195, miR-29c, miR-221/222 enhances TMZ resistance $(7,15-17,35,36)$. However, the function of miR-146b-5p in TMZ resistance has not been recognized. Previous studies demonstrated that miR-146b-5p was downregulated in recurrent GBM samples compared to that of primary ones, indicating its function in treatment resistance (37). Moreover, by binding to 3'-untranslated region of EGFR mRNA, miR-146b-5p suppresses the stemness and induces differentiation of GSCs, which also has a good part in TMZ resistance (21). In this study, we found that miR-146b-5p was downregulated in TMZ-resistant cells, and overexpression of miR-146b-5p conferred sensitivity to TMZ on GBM cells by targeting TRAF6. Although a previous study has reported that low expression of miR-146b-5p would enhance the glioma cell invasive capacity and correlate with poor prognosis for glioma patients (28), its role in TMZ resistance was first clarified. Since the miR-146b-5p also involves in modulating other target genes including MMP16 and EGFR $(18,38)$, its mechanisms of TMZ resistance need to be further investigated in the future.

TRAF6, as the E3 ubiquitin ligase, takes part in promoting oncogenesis. Overexpression of TRAF6 in mouse marrow cells leads to a myelodysplastic syndrome, which develops into a fatal acute myeloid leukemia eventually (39). It promotes proliferation and regulates apoptosis in breast cancer, osteosarcoma and lung adenocarcinoma cells $(25,26,40)$. Furthermore, overexpression of TRAF6 enhances the resistance of colon cancer cells to 5-fluorouracil and acute leukemia cells to bortezomib (27). In our study, we found that TRAF6, as a target gene of miR-146b-5p, not only played a substantial role in tumor proliferation ability, but also contributed TMZ resistance to GBM cells. Silencing of TRAF6 could mimic the antitumor effect of miR-146b-5p through reversing $\mathrm{TMZ}$ resistance and decreasing proliferation in GBM cells. Activation of some signaling pathways including $\mathrm{AKT}$ and $\mathrm{NF}-\kappa \mathrm{B}$ has been found to be involved in TRAF6-mediated oncogenesis $(26,28,41)$. Constitutive activation of $\mathrm{AKT} / \mathrm{NF}-\kappa \mathrm{B}$ pathway is frequently observed in different types of cancer and contributes to tumor progression, and chemoresistance (42-44). A previous study in metastatic melanoma cell line demonstrated that management of TMZ could activate the AKT/NF- $\kappa \mathrm{B}$, while inhibiting the activity of AKT and NF- $\kappa \mathrm{B}$ could sensitize cancer cells to TMZ (42). In order to confirm whether miR-146b-5p targeted TRAF6 overexpression contributes to $\mathrm{TMZ}$ resistance in glioblastoma by activating the $\mathrm{AKT} / \mathrm{NF}-\kappa \mathrm{B}$ pathway, signaling array experiments were conducted. Western blot analysis revealed that TR cells had a higher level of p-AKT and p-p65 which is an indicator of $\mathrm{NF}-\kappa \mathrm{B}$ activation compared to control group, and overexpression of miR-146b-5p or TRAF6 knockdown caused a marked reduction p-AKT and p-p65. It is well known that $\mathrm{AKT} / \mathrm{NF}-\kappa \mathrm{B}$ signaling pathway plays an essential role in regulation of autophagy which also contributes to TMZ resistance $(33,45)$. However, whether autophagy is regulated by miR-146b-5p targeted TRAF6 through AKT/NF- $\kappa$ B pathway in TMZ resistance is under investigation in our lab.

In conclusion, the results of the present study showed that TRAF6, the target gene of miR-146b-5p, was highly expressed in TR cells and associated with TMZ resistance. The integrated analyses depending on miRNAs and their related regulatory networks provide new insights into addressing TMZ resistance in GBM, and miR-146b-5p may serve as a novel and potential therapeutic agent for overcoming TMZ resistance in patients with gliomas.

\section{Acknowledgements}

This study was supported by the National Natural Science Foundation of China (no. 81402042), Shanghai Science and Technology (14140903400, 14YF1402600), the State Key Laboratory of Oncogenes and Related Genes (no. 90-14-01), the Shanghai Municipal Population and Family Planning Commission (2013SY024), the Key Specialty Construction Project and Science Technology Development Project of the Pudong Health and Family Commission of Shanghai (nos. PWZz2013-18 and PW2013A-19), and the Training Plan for Scientific Research of Renji Hospital (RJZZ13-021).

\section{References}

1. Stupp R, Mason WP, van den Bent MJ, Weller M, Fisher B, Taphoorn MJ, Belanger K, Brandes AA, Marosi C, Bogdahn U, et al; European Organisation for Research and Treatment of Cancer Brain Tumor and Radiotherapy Groups; National Cancer Institute of Canada Clinical Trials Group: Radiotherapy plus concomitant and adjuvant temozolomide for glioblastoma. N Engl J Med 352: 987-996, 2005.

2. Wen PY and Kesari S: Malignant gliomas in adults. N Engl J Med 359: 492-507, 2008. 
3. Walecki J, Tarasów E, Kubas B, CzemickiZ, Lewko J,Podgórski J, Sokól M and Grieb P: Hydrogen-1 MR spectroscopy of the peritumoral zone in patients with cerebral glioma: Assessment of the value of the method. Acad Radiol 10: 145-153, 2003.

4. Stupp R, Hegi ME, Mason WP, van den Bent MJ, Taphoorn MJ, Janzer RC, Ludwin SK, Allgeier A, Fisher B, Belanger K, et al; European Organisation for Research and Treatment of Cancer Brain Tumour and Radiation Oncology Groups; National Cancer Institute of Canada Clinical Trials Group: Effects of radiotherapy with concomitant and adjuvant temozolomide versus radiotherapy alone on survival in glioblastoma in a randomised phase III study: 5-year analysis of the EORTC-NCIC trial. Lancet Oncol 10: 459-466, 2009.

5. Stupp R and Hegi ME: Brain cancer in 2012: Molecular characterization leads the way. Nat Rev Clin Oncol 10: 69-70, 2013.

6. Knizhnik AV, Roos WP, Nikolova T, Quiros S, Tomaszowski KH, Christmann M and Kaina B: Survival and death strategies in glioma cells: Autophagy, senescence and apoptosis triggered by a single type of temozolomide-induced DNA damage. PLoS One 8: e55665, 2013.

7. Xiao S, Yang Z, Qiu X, Lv R, Liu J, Wu M, Liao Y and Liu Q: miR-29c contribute to glioma cells temozolomide sensitivity by targeting $\mathrm{O}^{6}$-methylguanine-DNA methyltransferases indirectely. Oncotarget 7: 50229-50238; Epub ahead of print, 2016.

8. Hermisson M, Klumpp A, Wick W, Wischhusen J, Nagel G, Roos W, Kaina B and Weller M: $\mathrm{O}^{6}$-methylguanine DNA methyltransferase and p53 status predict temozolomide sensitivity in human malignant glioma cells. J Neurochem 96: 766-776, 2006.

9. Zhang K, Wang XQ, Zhou B and Zhang L: The prognostic value of MGMT promoter methylation in glioblastoma multiforme: A meta-analysis. Fam Cancer 12: 449-458, 2013.

10. Wang H, Feng W, Lu Y, Li H, Xiang W, Chen Z, He M, Zhao L, Sun X, Lei B, et al: Expression of dynein, cytoplasmic 2, heavy chain 1 (DHC2) associated with glioblastoma cell resistance to temozolomide. Sci Rep 6: 28948, 2016.

11. Bartel DP: MicroRNAs: Genomics, biogenesis, mechanism, and function. Cell 116: 281-297, 2004.

12. Bartel DP: MicroRNAs: Target recognition and regulatory functions. Cell 136: 215-233, 2009.

13. Shukla GC, Singh J and Barik S: MicroRNAs: Processing, maturation, target recognition and regulatory functions. Mol Cell Pharmacol 3: 83-92, 2011.

14. Hummel R, Hussey DJ and Haier J: MicroRNAs: Predictors and modifiers of chemo- and radiotherapy in different tumour types. Eur J Cancer 46: 298-311, 2010.

15. Wei J, Qi X, Zhan Q, Zhou D, Yan Q, Wang Y, Mo L, Wan Y, Xie D, Xie J, et al: miR-20a mediates temozolomide-resistance in glioblastoma cells via negatively regulating LRIG1 expression. Biomed Pharmacother 71: 112-118, 2015.

16. Ujifuku K, Mitsutake N, Takakura S, Matsuse M, Saenko V, Suzuki K, Hayashi K, Matsuo T, Kamada K, Nagata I, et al: miR-195, miR-455-3p and miR-10a $(*)$ are implicated in acquired temozolomide resistance in glioblastoma multiforme cells. Cancer Lett 296: 241-248, 2010.

17. Wang J, Sai K, Chen FR and Chen ZP: miR-181b modulates glioma cell sensitivity to temozolomide by targeting MEK1. Cancer Chemother Pharmacol 72: 147-158, 2013.

18. Li Y, Wang Y, Yu L, Sun C, Cheng D, Yu S, Wang Q, Yan Y, Kang C, Jin S, et al: miR-146b-5p inhibits glioma migration and invasion by targeting MMP16. Cancer Lett 339: 260-269, 2013.

19. van Thuijl HF, Scheinin I, Sie D, Alentorn A, van Essen HF, Cordes M, Fleischeuer R, Gijtenbeek AM, Beute G, van den Brink WA, et al: Spatial and temporal evolution of distal 10q deletion, a prognostically unfavorable event in diffuse low-grade gliomas. Genome Biol 15: 471, 2014

20. Weller M, Weber RG, Willscher E, Riehmer V, Hentschel B, Kreuz M, Felsberg J, Beyer U, Löffler-Wirth H, Kaulich K, et al: Molecular classification of diffuse cerebral WHO grade II/III gliomas using genome- and transcriptome-wide profiling improves stratification of prognostically distinct patient groups. Acta Neuropathol 129: 679-693, 2015.

21. Yang W, Yu H, Shen Y, Liu Y, Yang Z and Sun T: MiR-146b-5p overexpression attenuates stemness and radioresistance of glioma stem cells by targeting HuR/lincRNA-p $21 / \beta$-catenin pathway. Oncotarget 7: 41505-41526, 2016.
22. Liu H, Tamashiro S, Baritaki S, Penichet M, Yu Y, Chen H, Berenson $\mathrm{J}$ and Bonavida B: TRAF6 activation in multiple myeloma: A potential therapeutic target. Clin Lymphoma Myeloma Leuk 12: 155-163, 2012.

23. Inoue J, Gohda $\mathrm{J}$ and Akiyama $\mathrm{T}$ : Characteristics and biological functions of TRAF6. Adv Exp Med Biol 597: 72-79, 2007.

24. Chen ZJ: Ubiquitination in signaling to and activation of IKK Immunol Rev 246: 95-106, 2012.

25. Lin Y, Qiu Y, Xu C, Liu Q, Peng B, Kaufmann GF, Chen X, Lan B, Wei C, Lu D, et al: Functional role of asparaginyl endopeptidase ubiquitination by TRAF6 in tumor invasion and metastasis. J Natl Cancer Inst 106: dju012, 2014.

26. Starczynowski DT, Lockwood WW, Deléhouzée S, Chari R, Wegrzyn J, Fuller M, Tsao MS, Lam S, Gazdar AF, Lam WL, et al: TRAF6 is an amplified oncogene bridging the RAS and NF- $\kappa \mathrm{B}$ pathways in human lung cancer. J Clin Invest 121: 4095-4105, 2011.

27. Sun H, Li X, Fan L, Wu G, Li M and Fang J: TRAF6 is upregulated in colon cancer and promotes proliferation of colon cancer cells. Int J Biochem Cell Biol 53: 195-201, 2014.

28. Liu J, Xu J, Li H, Sun C, Yu L, Li Y, Shi C, Zhou X, Bian X, Ping Y, et al: miR-146b-5p functions as a tumor suppressor by targeting TRAF6 and predicts the prognosis of human gliomas. Oncotarget 6: 29129-29142, 2015.

29. Yi GZ, Liu YW, Xiang W, Wang H, Chen ZY, Xie SD and Qi ST: Akt and $\beta$-catenin contribute to TMZ resistance and EMT of MGMT negative malignant glioma cell line. J Neurol Sci 367: 101-106, 2016.

30. Kim SH, Joshi K, Ezhilarasan R, Myers TR, Siu J, Gu C, Nakano-Okuno M, Taylor D, Minata M, Sulman EP, et al: EZH2 protects glioma stem cells from radiation-induced cell death in a MELK/FOXM1-dependent manner. Stem Cell Rep 4: 226-238, 2015.

31. Shi L, Fei X, Wang Z and You Y: PI3K inhibitor combined with miR-125b inhibitor sensitize TMZ-induced anti-glioma stem cancer effects through inactivation of Wnt/ $\beta$-catenin signaling pathway. In Vitro Cell Dev Biol Anim 51: 1047-1055, 2015.

32. Fukushima T, Takeshima $\mathrm{H}$ and Kataoka $\mathrm{H}$ : Anti-glioma therapy with temozolomide and status of the DNA-repair gene MGMT Anticancer Res 29: 4845-4854, 2009.

33. Yan Y, Xu Z, Dai S, Qian L, Sun L and Gong Z: Targeting autophagy to sensitive glioma to temozolomide treatment. J Exp Clin Cancer Res 35: 23; 2016.

34. Sun YC, Wang J, Guo CC, Sai K, Wang J, Chen FR, Yang QY, Chen YS, Wang J, To TS, et al: MiR-181b sensitizes glioma cells to teniposide by targeting MDM2. BMC Cancer 14: 611, 2014.

35. Comincini S, Allavena G, Palumbo S, Morini M, Durando F, Angeletti F, Pirtoli L and Miracco C: microRNA-17 regulates the expression of ATG7 and modulates the autophagy process, improving the sensitivity to temozolomide and low-dose ionizing radiation treatments in human glioblastoma cells. Cancer Biol Ther 14: 574-586, 2013.

36. Chen L, Zhang J, Han L, Zhang A, Zhang C, Zheng Y, Jiang T, $\mathrm{Pu}$ P, Jiang C and Kang C: Downregulation of miR-221/222 sensitizes glioma cells to temozolomide by regulating apoptosis independently of p53 status. Oncol Rep 27: 854-860, 2012.

37. Bo LJ, Wei B, Li ZH, Wang ZF, Gao Z and Miao Z: Bioinformatics analysis of miRNA expression profile between primary and recurrent glioblastoma. Eur Rev Med Pharmacol Sci 19: 3579-3586, 2015

38. Katakowski M, Zheng X, Jiang F, Rogers T, Szalad A and Chopp M: MiR-146b-5p suppresses EGFR expression and reduces in vitro migration and invasion of glioma. Cancer Invest 28: 1024-1030, 2010

39. Starczynowski DT, Kuchenbauer F, Argiropoulos B, Sung S, Morin R, Muranyi A, Hirst M, Hogge D, Marra M, Wells RA, et al: Identification of miR-145 and miR-146a as mediators of the 5q- syndrome phenotype. Nat Med 16: 49-58, 2010.

40. Meng Q, Zheng M, Liu H, Song C, Zhang W, Yan J, Qin L and Liu X: TRAF6 regulates proliferation, apoptosis, and invasion of osteosarcoma cell. Mol Cell Biochem 371: 177-186, 2012.

41. Han F, Zhang L, Qiu W and Yi X: TRAF6 promotes the invasion and metastasis and predicts a poor prognosis in gastric cancer. Pathol Res Pract 212: 31-37, 2016.

42. Caporali S, Levati L, Graziani G, Muzi A, Atzori MG, Bonmassar E, Palmieri G, Ascierto PA and D'Atri S: NF- $\kappa B$ is activated in response to temozolomide in an AKT-dependent manner and confers protection against the growth suppressive effect of the drug. J Transl Med 10: 252, 2012. 
43. Liu JF, Tsao YT and Hou CH: Fractalkine/CX3CL1 induced intercellular adhesion molecule-1-dependent tumor metastasis through the CX3CR1/PI3K/Akt/NF- $\mathrm{BB}$ pathway in human osteosarcoma. Oncotarget; Aug 12, 2016 (Epub ahead of print). doi: 10.18632/oncotarget.11250.

44. Zou W, Ma X, Hua W, Chen B and Cai G: Caveolin-1 mediates chemoresistance in cisplatin-resistant ovarian cancer cells by targeting apoptosis through the Notch-1/Akt/NF- $\kappa \mathrm{B}$ pathway. Oncol Rep 34: 3256-3263, 2015.

45. Barré B and Perkins ND: The Skp2 promoter integrates signaling through the NF-kappaB, p53, and Akt/GSK3beta pathways to regulate autophagy and apoptosis. Mol Cell 38 $524-538,2010$ 